\title{
SET knockdown attenuated phenotype modulation and calcium channel associated markers of airway smooth muscle cells in asthmatic mice
}

\author{
Jie Li", Qi He ${ }^{\#}$, Lingwei Wang, Dandan Chen, Chen Qiu, Peng Xu, Yongzhen Lu, Yuwei Zeng, \\ Rongchang Chen
}

Key Laboratory of Shenzhen Respiratory Disease, Shenzhen Institute of Respiratory Disease, Shenzhen People's Hospital (The First Affiliated Hospital of Southern University of Science and Technology, The Second Clinical Medical College of Jinan University), Shenzhen, China

Contributions: (I) Conception and design: J Li, Q He, R Chen; (II) Administrative support: J Li, R Chen; (III) Provision of study materials or patients: All authors; (IV) Collection and assembly of data: J Li, Q He, L Wang, D Chen, P Xu, Y Lu, Y Zeng; (V) Data analysis and interpretation: J Li, Q He, L Wang, D Chen, P Xu, Y Lu, Y Zeng; (VI) Manuscript writing: All authors; (VII) Final approval of manuscript: All authors.

\#These authors contributed equally to this work.

Correspondence to: Jie Li; Rongchang Chen. Key Laboratory of Shenzhen Respiratory Disease, Shenzhen Institute of Respiratory Disease, Shenzhen People's Hospital (The First Affiliated Hospital of Southern University of Science and Technology, The Second Clinical Medical College of Jinan University), Shenzhen, China. Email: ljszry2018@126.com; chenrc@vip.163.com.

Background: Dysfunctional phenotype modulation and calcium channels in airway smooth muscle cells (ASMCs) are important characteristics of airway remodeling in chronic asthma. However, the mechanisms underlying these pathological processes remain unclear. SET (I2PP2A, inhibitor-2 of protein phosphatase 2A) has many significant functions and is involved in various physiological and pathological processes. This study aimed to determine the function of SET in chronic asthma.

Methods: BALB/c mice were sensitized by ovalbumin injection and repeated inhalation of ovalbumin. The Penh value was measured using the Buxco whole body plethysmography system. A short hairpin RNA of the SET gene was designed and transfected into ASMCs derived from asthmatic mice. Flow cytometry of Annexin-V/propidium iodide staining was used for evaluating cell apoptosis. Western blot was adopted to measure the expression levels of ASMCs phenotype modulation markers and calcium channel-associated proteins.

Results: The results showed that shRNA targeting SET significantly decreased the expression of SET, and enhanced the apoptosis of ASMCs. SET knockdown promoted the expression of contractile phenotype markers such as $\alpha$-SMA (alpha smooth muscle Actin), SM-MHC (smooth muscle Myosin heavy chain), and calponin, and inhibited the expression of synthetic phenotype markers including vimentin and CD44. The expression of the calcium channel-related proteins STIM1 (Stromal interaction molecule 1) and Orai1 were also inhibited after SET knockdown.

Conclusions: These data demonstrated that SET participated in the development of airway dysfunction in asthma, suggesting that the silencing of SET may be a new therapeutic target for the treatment of asthma patients.

Keywords: Asthma; airway smooth muscle cells (ASMCs); SET; phenotype modulation; calcium channel

Submitted Dec 14, 2020. Accepted for publication Apr 15, 2021.

doi: $10.21037 /$ atm-21-573

View this article at: http://dx.doi.org/10.21037/atm-21-573 


\section{Introduction}

Asthma is a common chronic respiratory disease that affects more than 300 million people (1). The clinical symptoms of patients include airway inflammation, remodeling, and airway hyper-responsiveness (AHR) $(2,3)$. In recent years, numerous investigations have revealed that airway remodeling is a common feature in biopsy studies of all types of asthma $(4,5)$. The abnormal change in airway smooth muscle (ASM) is a significant pathological characteristic of airway remodeling. Airway smooth muscle cells (ASMCs) are instrumental to airway remodeling due to their multiple roles in asthma (6).

ASMCs have two phenotypes include contractile phenotype and synthetic phenotype. It is normally contractile phenotype and the phenotype modulation of ASMCs may contribute to the onset of asthma (7). It has been reported that ASMCs exposed to a series of mitogens resulted in reversible changes from a contractile phenotype to a synthetic phenotype (8), which characterized with strong proliferation and division ability, while significantly increase in organelles those can synthesize protein and lipid. Airway inflammatory diseases such as asthma and COPD can cause phenotypic transformation of ASMCs. ASMCs calcium channel dysfunction is another important feature of asthma and is also associated with airway remodeling. The membrane of ASMCs is rich in store-operated calcium channels (SOCCs) and voltage-dependent calcium channels (VDCCs). ASM is a key cell type in contractility and remodeling of asthma, abnormal activation of the calcium channels leads to increased intracellular calcium and activates excitation-contraction coupling, which promotes ASMCs proliferation and makes ASMCs more sensitive to stimuli and causes airway remodeling $(9,10)$. In the ASMCs, androgen receptor is responsible for the bronchodilator effect and its expression is increased in female with asthma. And in female asthmatic patients, estrogen receptors on the cell membrane interacting with estrogen more quickly following produce biological effects. Then estrogen receptors are activated and promoted intracellular calcium concentration. So we chose female mice as animal model in our study.

A study of acute undifferentiated leukemia in 1992 first identified the SET protein, also termed TAF-1 $\beta$ and I2PP2A (11). The SET protein has many important functions due to its highly complex structure and evolution mechanism. SET is overexpressed in many malignancies, such as testicular cancer (12), lung tumors (13), tumors of the head and neck (14), breast cancer (15), and prostate cancer (16). It has been demonstrated that SET is involved in tumorigenesis due to the inhibition of the tumor suppressor gene PP2A (17). SET mediates many signal transduction pathways, including SET/ERK and SET/ p38MAPK, and further affects the pathophysiological processes of ASMCs. However, the function of SET in the pathogenesis of asthma is not entirely understood. There have been no reports on the study of SET in asthma. We have made a pioneering research on the relationship between SET and ASMCs phenotypic transformation and calcium channels in asthma, hoping to provide basis for the research in asthma.

Therefore, this work aimed to investigate the development of ASM phenotypic transformation, calcium channel dysfunction, and the involvement of SET in these 2 physiological processes. The influence of SET knockdown with lentivirus-mediated shRNA on airway remodeling was also investigated. Our results provide a theoretical and experimental basis for potential asthma treatments.

We present the following article in accordance with the ARRIVE reporting checklist (available at http://dx.doi. org/10.21037/atm-21-573).

\section{Methods}

\section{Chemical and reagents}

The 293T cell line was obtained from the Cell Resource Center of Shanghai Institutes for Biological Science (Chinese Academy of Science, China). Antibodies against a-SMA, SM-MHC, calponin, vimentin, CD44, STIM1, PI3K, and calmodulin were obtained from Abcam (Abcam, Cambridge, UK). The mouse monoclonal antibodies against SET and $\beta$-actin were purchased from Santa Cruz Biotechnology (Santa Cruz, CA, USA). The secondary antibodies were purchased from Thermo Fisher (Thermo Fisher, USA). The pLVX-shRNA1 expression vector was obtained from Clonetech (Mountain View, CA, USA).

\section{Mice}

Six-week-old female BALB/C mice (obtained from the center of Medical Experiment Animal of Guangdong Province, China) were used for animal experiments. Animal experiments are approved by the Animal Care and Use Committee of the Experimental Animal Center at Shenzhen Center for Disease Control and Prevention. 
Animal experiment were performed in accordance with the Principles of Laboratory Animal Care (NIH publication No. 85-23, revised 1985) and the Regulations of the Animal Care and Use Committee of the Experimental Animal Center at Shenzhen Center for Disease Control and Prevention. All efforts were made to minimize suffering of the animals and to reduce the number of mice used.

\section{Animal sensitization and airway challenge}

The BALB/C mice group $(n=10)$ was sensitized and challenged. Sensitization was induced by intraperitoneal injection of $20 \mu \mathrm{g}$ ovalbumin (OVA, grade V) mixed in $50 \mu \mathrm{L}$ of $30 \mathrm{mg} / \mathrm{mL}$ Inject ${ }^{\circledR}$ Alum on days $0,14,28$, and 42 . Then, challenges were induced by $5 \%$ grade II OVA 3 times per week and 30 min each time from day 2. The control group $(\mathrm{n}=10)$ was treated with normal saline in a similar manner.

\section{Non-invasive measurement of Penb}

We used the Buxco whole body plethysmography (WBP) system to record respiratory pressure curves (Buxco electronics, St. Paul, MN, USA) for evaluating Penh $24 \mathrm{~h}$ after the last aerosol exposure in response to inhaled methacholine (mAch) (0-100 mg/mL) in conscious unrestrained mice. mAch was aerosolized for $3 \mathrm{~min}$, and we recorded the readings every $5 \mathrm{~min}$ after each nebulization was averaged.

\section{Lung bistopatbology}

Right upper lobes and right middle lobes were harvested for histopathological analysis. Tissues were fixed in $4 \%$ formaldehyde for $24 \mathrm{~h}$, then paraffin-embedded and cut into $5 \mu \mathrm{m}$ sections for staining. Hematoxylin and eosin (H\&E) was used for assessing the thickness of the smooth muscle layer. Morphometry was assessed by blinded individuals.

\section{Primary ASMCs culture from mice}

Primary ASMCs were isolated by a modified protocol according to a previous study (18). We entirely separated the trachea and lungs, carefully removed the extraneous membranes and connective tissues, and cut the tissue into cross-sections of 2-3 mm. The tissue was placed in a culture flask and immersed in Dulbecco's modified Eagle medium (DMEM) containing 20\% fetal bovine serum, $4 \mathrm{mM}$ glutamine, and $1 \%$ penicillin-streptomycin. The cultured specimens were incubated in a $5 \% \mathrm{CO}_{2}$ incubator at $37{ }^{\circ} \mathrm{C}$ until $80 \%$ confluent. We obtained the desired cells which could be used for experiments after purification.

\section{Western blot analysis}

We extracted protein from lung tissues and cells with lysis buffer (Beyotime Biotech, China) based on our previous experience. The protein content was measured with a BCA assay. Proteins were then separated using 10\% SDSPAGE and electrically transferred onto PVDF membranes (Millipore, MA, USA). The membranes were incubated with TBST buffer with $5 \%$ milk and then probed with antibodies against SET, $\alpha$-SMA, SM-MHC, calponin, vimentin, CD44, STIM1, Orai1, and $\beta$-actin. Horseradish peroxidase-conjugated secondary antibodies were used to detect blots. The protein bands were then incubated with Supersignal ${ }^{\circledR}$ West Dura Extended Duration Substrate (Thermo Scientific, USA) and visualized with the ECLTM western blotting detection system (ImageQuant TM RT, GE Healthcare).

\section{SET immunobistochemistry}

Immunohistochemical staining was carried out according to the instructions Briefly, $5 \mu \mathrm{m}$ sections of paraffin-embedded lung tissues were de-paraffinized, dehydrated, and incubated in a $10 \%$ hydrogen peroxide buffer for $10 \mathrm{~min}$ to eliminate endogenous peroxidase activity. The sections were then incubated with SET antibody (1:200) solution overnight at $4{ }^{\circ} \mathrm{C}$. Anti-mouse secondary antibody with horseradish peroxidase polymer was then applied. The sections were stained with the DAB plus kit and analyzed using ImagePro Plus software. Specific labeling was unnoticed in sections lacking the primary antibody.

\section{Generation of lentivirus shRNA SET knockdown ASMCs}

The siRNA oligonucleotides targeting mouse SET and human SET were purchased from Sangon Biotech (Shanghai, China) and cloned into pLVX-shRNA1 vectors. We used the blank plasmid as a control. The pseudotyped lentiviruses were produced in 293T cells by co-transfection with siRNA plasmids and blank plasmids through the lentivirus transfection packaging kit. ASMCs were seeded in 6-well plates, and transfected with lentiviruses encoding SET shRNA using $4.0 \mu \mathrm{g} / \mathrm{mL}$ polybrene. Then, they were treated with medium containing $2.0 \mu \mathrm{g} / \mathrm{mL}$ puromycin for 15 days. Western blot analysis determined the efficiency of 
the SET knockdown.

\section{Flow cytometric analysis of apoptosis}

Apoptosis of ASMCs was detected by staining with the Annexin V-FITC cell apoptosis detection kit (Nanjing, keygen, Biotech Co., Ltd, China). Cells were harvested and incubated in diluted binding buffer, before adding $5 \mu \mathrm{L}$ propidium iodide (PI, $20 \mu \mathrm{g} / \mathrm{mL}$ ) for $15 \mathrm{~min}$ in the dark. Apoptosis was measured using a flow cytometer (BD, Franklin Lakes, NJ, USA).

\section{Statistical analysis}

All data were expressed as mean \pm SD and analyzed using SPSS 18.0 software (SPSS Inc., Chicago, USA). Student's $t$-test and ANOVA were used for analysis, and $\mathrm{P}<0.05$ was considered statistically significant.

\section{Results}

Airway remodeling and Penb in chronic OVA-sensitized mice

To investigate whether OVA-sensitized mice developed airway remodeling, AHR and the cross-sectional areas of smooth muscle layers were measured. There were no signs of asthma in the normal group, while mice in the asthma group had sensitivity irregular respiratory rhythm, sneeze, and cyanosis of the lips. As shown in Figure $1 A$ the Penh value of the asthma group was significantly enhanced compared to the normal group. From the histological analysis (Figure $1 B$ ), there was significant inflammatory change in the lung mucosal and submucosal areas. In addition, the airway walls were comparatively thicker in the asthma group than in the normal group (Figure 1C).

\section{Expression of SET is increased in asthma}

To assess the expression of SET, immunohistochemical analysis was performed. Representative images of the staining are shown in Figure $1 D$. Through the immunohistochemistry results, it was observed that positive staining of SET was weak in normal tissues, while the SET level was increased significantly in the asthma group. Lung tissues of mice were also analyzed for SET using western blot. As shown in Figure 1E, the results demonstrated that there was a significant increase in the protein expression of
SET in the asthma group compared to mice in the normal group.

\section{Construction of stable SET siRNA ASMCs}

To confirm the efficiency of SET shRNA with RNA interference in asthmatic mouse ASMCs, SET protein was evaluated with western blot analysis. Figure $2 A, B$ indicated that transfection with psiRNA-SET inhibited SET expression effectively after 15 days of puromycin selection. In contrast, the transfection of the empty vector (pLVX) did not obviously influence the expression of SET. The data indicated the successful construction of stable SET knockdown ASMCs.

\section{Knockdown of SET expression increases cell apoptosis}

To evaluate the effect of SET inhibition on the apoptosis of ASMCs, cell apoptosis was measured by flow cytometry. Figure $3 A, B$ suggested that the cell apoptosis of SET knockdown ASMCs was increased compared to the control cells. There were no noticeable differences in the cell apoptosis of ASMCs between the empty group and the control group, which suggested that knockdown of SET obviously enhanced the apoptosis of ASMCs.

\section{Effects of SET knockdown on protein levels of phenotype switching markers}

To further confirm the effects of SET knockdown on the modulation of phenotype, western blot determined the protein levels of contractile phenotype and synthetic phenotype markers. As shown in Figure 4, SET knockdown ASMCs had increased contractile phenotype protein expression, including $\alpha$-SMA, SM-MHC, and calponin. In contrast, the expression of synthetic phenotype proteins such as vimentin and CD44 decreased in SET knockdown ASMCs. There was no significant difference between the control cells and the empty vector cells.

\section{Regulation of ASMCs calcium channels by knockdown of SET}

Due to the importance of the abnormal activation of calcium channels in the airway remodeling process of asthma, a thorough exploration was performed on the effects of SET knockdown on SOCC-related proteins in ASMCs. As shown in Figure 5, the expression of STIM1 
A

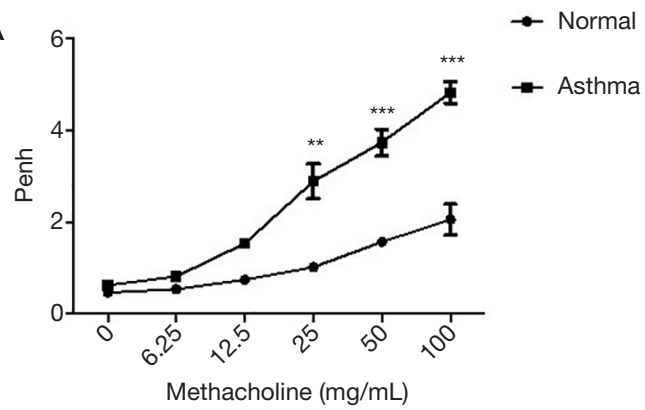

C

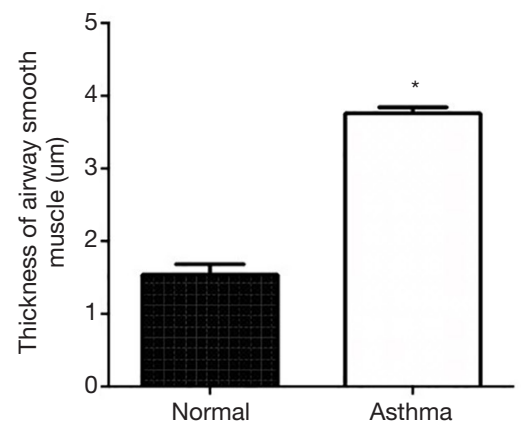

$\mathrm{E}$

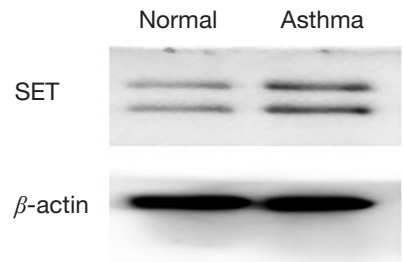

B
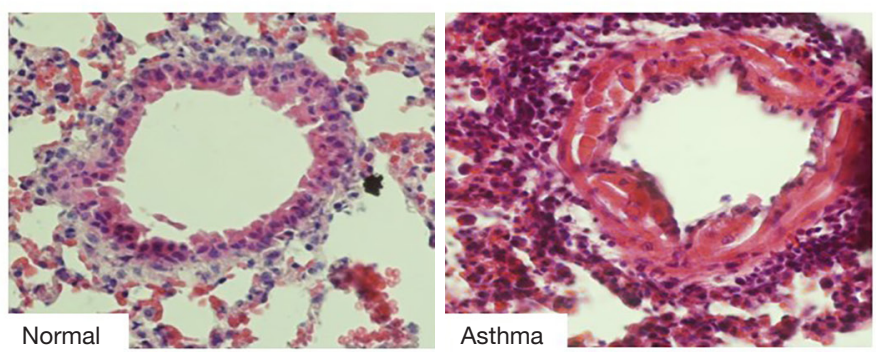

D
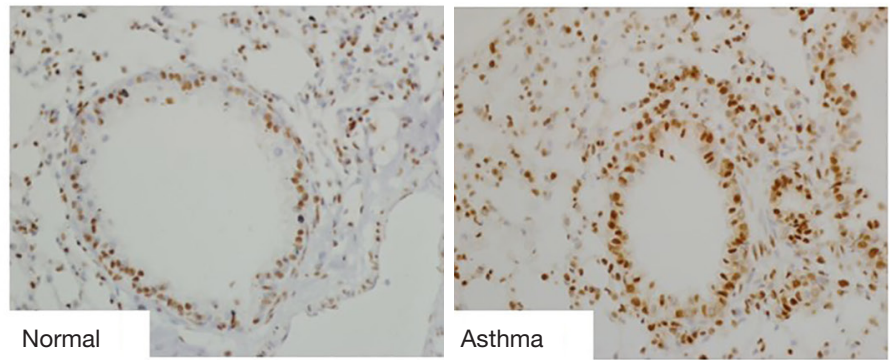

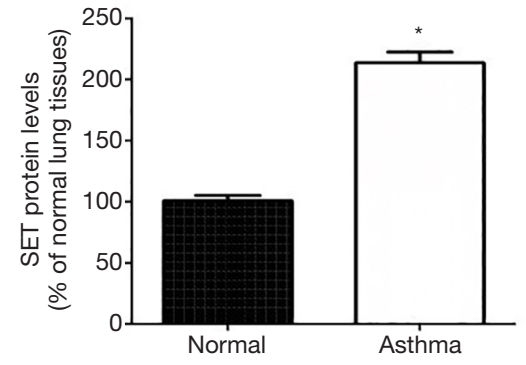

Figure 1 Validation of chronic OVA-challenged asthmatic models and the level of SET protein. (A) Penh in the OVA-challenged group (abbreviated as asthma group) and the normal group. ${ }^{* *} \mathrm{P}<0.01,{ }^{* * *} \mathrm{P}<0.001$, compared with the normal group $(\mathrm{n}=10)$. (B) Representative images of lung sections from each group using H\&E $(\times 400)$. (C) Smooth muscle layers thickened in the asthma group. ${ }^{*} \mathrm{P}<0.05$, compared with the normal group. (D) Representative images of lung sections from the asthma group and the normal group using IHC $(\times 100)$. (E) The protein expression of SET in the asthma group and the normal group. ${ }^{*} \mathrm{P}<0.05$, compared with the normal group $(\mathrm{n}=10)$.

and Orai1 were significantly suppressed in SET knockdown ASMCs compared to control cells, whereas similar expression levels were found between the control cells and the empty vector cells.

\section{Discussion}

Airway remodeling is an important characteristic of asthma (19), which was confirmed by our animal experiments. We found that SET was overexpressed in the lung tissues of the asthma group. It has been reported that SET affects cell apoptosis, chromatin remodeling, and DNA repair (20,21). SET has also been shown to be involved in the development of various diseases, including tumors (22), Alzheimer's disease, canine melanoma, and acute myeloid leukemia (23). Our findings, together with the diverse functions of SET, suggest that the overexpression of SET has a significant effect on asthma.

There are many researches on ASMCs phenotype modulation of asthma and it's clear that the process is affected by many factors, but the specific regulatory mechanism is not clear. The modulation of ASMCs phenotypes can 
A

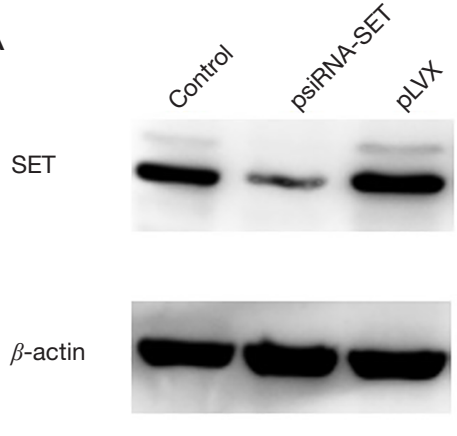

B

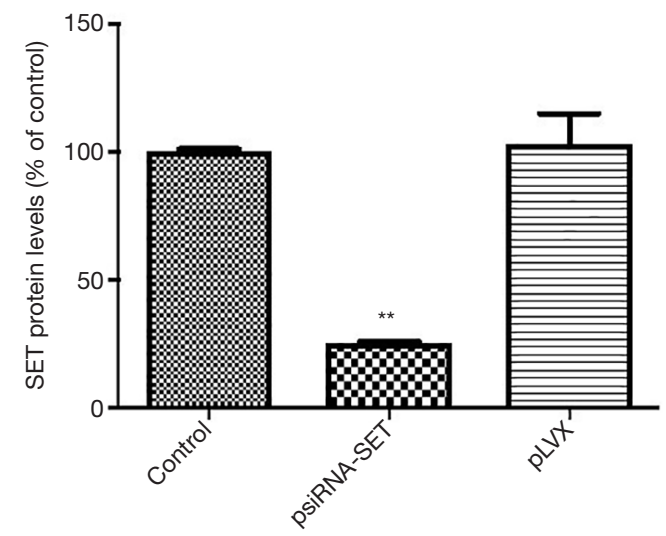

Figure 2 The expression of SET is downregulated by siRNA in ASMCSs. (A) Representative images of western blot. (B) Quantification of the SET bands. The data represent the mean $\pm \mathrm{SD}(\mathrm{n} \geq 3){ }^{* *} \mathrm{P}<0.01$, vs. control cells. ASMCS, airway smooth muscle cell.

A

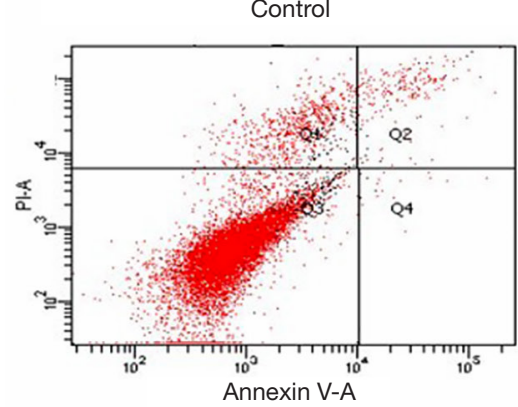

B

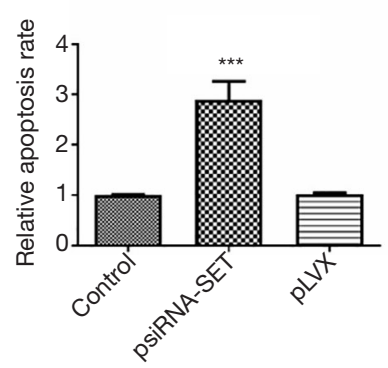

psiRNA-SET

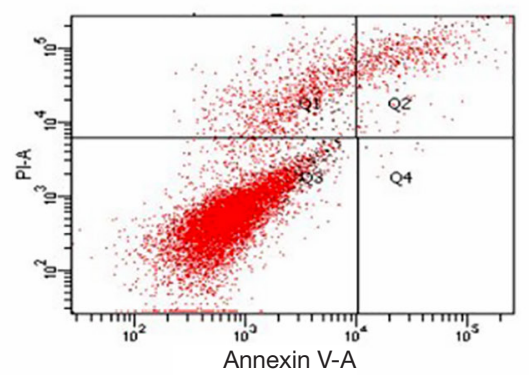

pLVX

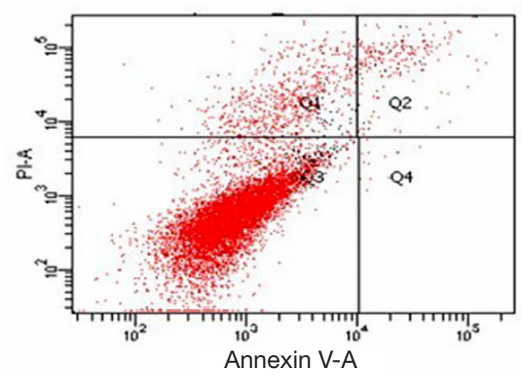

Figure 3 Effects of SET knockdown on cell apoptosis in ASMCs. (A) Representative Annexin-V/PI flow cytometry data of the different groups of ASMCs. (B) The quantified flow cytometry data. The data represent the mean $\pm \mathrm{SD}\left(\mathrm{n} \geq 3\right.$ ). ${ }^{* *} \mathrm{P}<0.001, v s$. control cells. ASMCs, airway smooth muscle cell.

affect asthma pathology, and is defined as the switch from a contractile phenotype to a synthetic phenotype, along with an array of functional changes that are yet to be understood (7). In this study, $\alpha$-SMA, SM-MHC, and calponin were selected as representative contractile phenotype markers consistent with Ueki et al.'s research (24), and their protein expression levels were detected. Our results indicated that contractile phenotype proteins were increased due to SET knockdown.
On the other hand, vimentin and CD44 were selected as synthetic phenotype markers according to Halayko et al.'s report (25), and it was found that the expression of these proteins decreased after SET knockdown. These results highlight the abnormal switching of ASMCs phenotypes in asthma, which might be attenuated by SET knockdown.

Cytoplasmic $\mathrm{Ca}^{2+}$ is a critical intracellular signal in many cell types and mostly originates from SOCCs and 
A
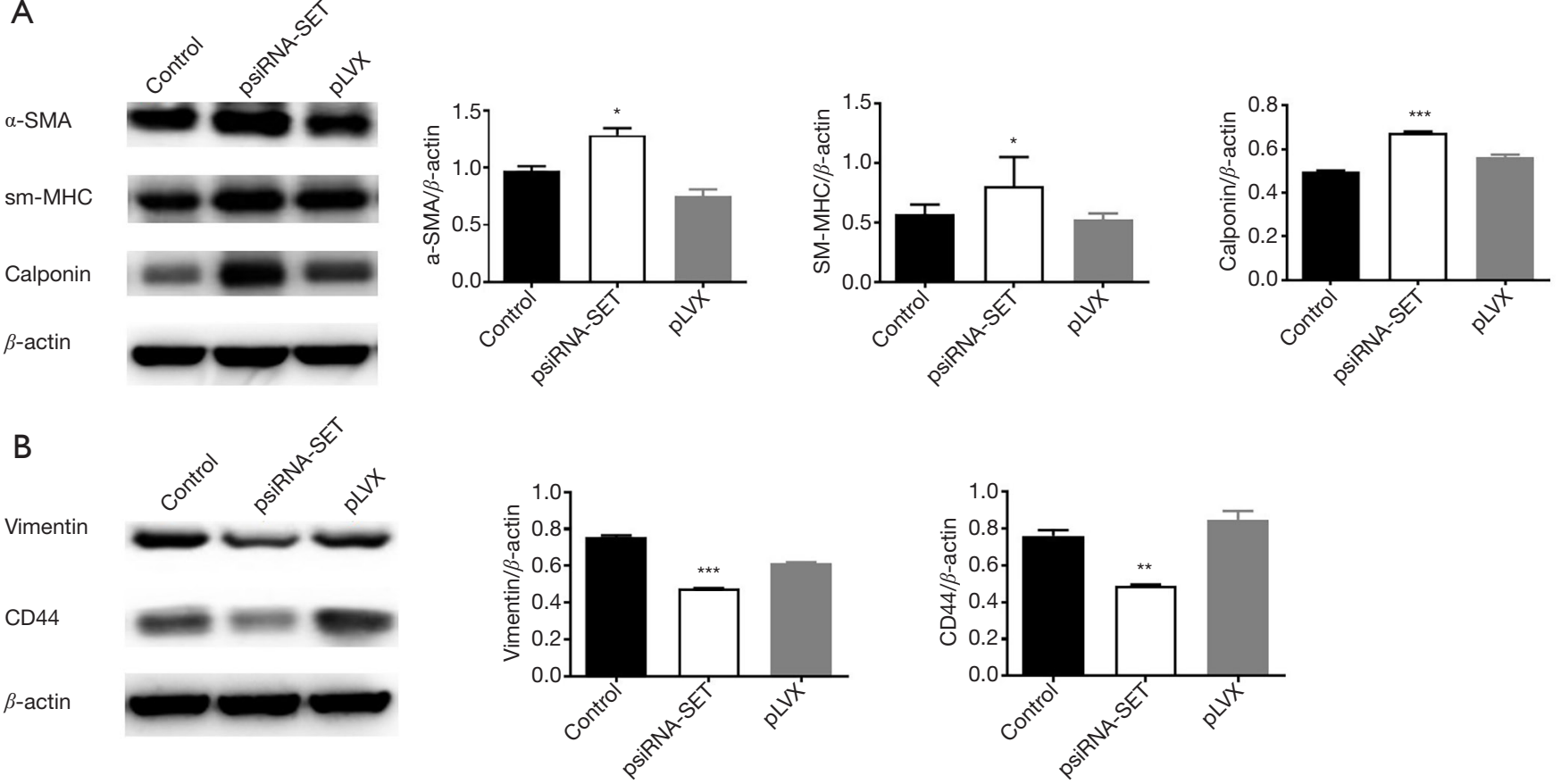

Figure 4 Knockdown of SET attenuates the phenotype modulation of ASMCs. The levels of contractile phenotype proteins (A) and synthetic phenotype proteins (B) were validated by western blotting. The data represent the mean $\pm \mathrm{SD}(\mathrm{n} \geq 3)$. ${ }^{*} \mathrm{P}<0.05$, ${ }^{* *} \mathrm{P}<0.01$, ${ }^{* * *} \mathrm{P}<0.001, v s$. control cells. ASMCs, airway smooth muscle cell.

STIM1
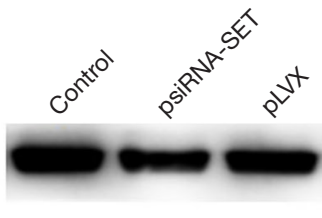

Orai1

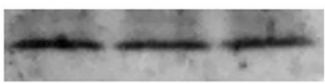

$\beta$-actin

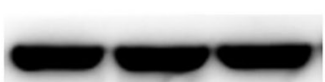

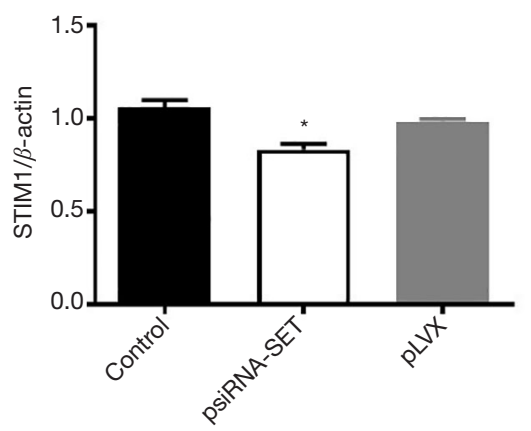

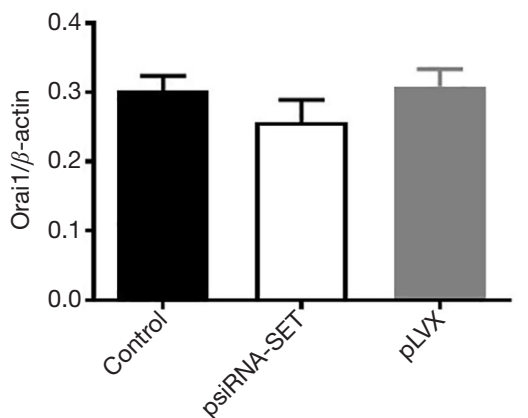

Figure 5 Knockdown of SET prevents the expression of calcium channel proteins. The levels of calcium channel proteins were validated by western blotting. The data represent the mean $\pm \mathrm{SD}(\mathrm{n} \geq 3)$. ${ }^{*} \mathrm{P}<0.05$, compared with the control cells.

VDCCs in the plasma membrane (26). The adjustment of intracellular $\mathrm{Ca}^{2+}$ concentrations in ASMCs is essential for airway contraction in asthma (27). Ohga et al. demonstrated that an inhibitor of SOCC could attenuate OVA-induced bronchoconstriction in guinea pigs (28). Nearly 2 decades ago, it was discovered that STIM1 and Orail were the molecular basis of SOCCs (29). Many studies have demonstrated increases in STIM1 and Orail in ASMCs derived from asthmatic mice $(9,30,31)$. The knockdown of STIM1 or Orai1 with siRNA resulted in a reduction of SOCCs in response to storage depletion of thapsigargin or cyclopiazonic acid in human ASMCs $(30,32)$. Our study indicated that the expression of STIM1 and Orai1 simultaneously decreased in SET knockdown ASMCs. This is consistent with evidence demonstrating that silencing of the $\beta 1$ integrin gene attenuated the increase of STIM1 and 
Orail in asthma (9).

Apoptosis is the spontaneous and orderly death of cells controlled by genes in order to maintain homeostasis. Inhibiting the apoptosis of normal cells or promoting the apoptosis of pathological cells has profound significance for humans. Previous in vivo and in vitro studies revealed that the apoptosis of ASMCs in asthma decreased clearance (33). Annexin V/PI double staining has evolved into an established way of detecting cell apoptosis because of its accuracy (34). Our study found that apoptosis was accelerated to some extent in asthma ASMCs after SET knockdown. This is in line with the effects of $\beta 1$-integrin shRNA on the cell apoptosis of ASMCs reported by Shi et al.'s team (18).

In conclusion, the current study demonstrated that SET played a role in ASM phenotype modulation and intracellular $\mathrm{Ca}^{2+}$ in the process of asthma, highlighting it as a therapeutic target with the potential to attenuate airway remodeling in chronic asthma.

\section{Acknowledgments}

Funding: This work was supported by the NSFC (No. 81770028), the Natural Science Foundation of Guangdong (2018A030310011), the Key Laboratory of Shenzhen Respiratory Disease (ZDSYS201504301616234), the Project of Shenzhen Basic Research Plan (JCYJ20170307095633450), and the Clinical research of Shenzhen Municipal Health and Family Planning Commission (SZLY2017024). This work also was supported by special funding for high-level disciplines from the Shenzhen Institute of Respiratory Diseases.

\section{Footnote}

Reporting Checklist: The authors have completed the ARRIVE reporting checklist. Available at http://dx.doi. org/10.21037/atm-21-573

Data Sharing Statement: Available at http://dx.doi. org/10.21037/atm-21-573

Conflicts of Interest: All authors have completed the ICMJE uniform disclosure form (available at http://dx.doi. org/10.21037/atm-21-573). The authors have no conflicts of interest to declare.

Ethical Statement: The authors are accountable for all aspects of the work in ensuring that questions related to the accuracy or integrity of any part of the work are appropriately investigated and resolved. Animal experiments are approved by the Animal Care and Use Committee of the Experimental Animal Center at Shenzhen Center for Disease Control and Prevention. Animal experiment were performed in accordance with the Principles of Laboratory Animal Care (NIH publication No. 85-23, revised 1985) and the Regulations of the Animal Care and Use Committee of the Experimental Animal Center at Shenzhen Center for Disease Control and Prevention.

Open Access Statement: This is an Open Access article distributed in accordance with the Creative Commons Attribution-NonCommercial-NoDerivs 4.0 International License (CC BY-NC-ND 4.0), which permits the noncommercial replication and distribution of the article with the strict proviso that no changes or edits are made and the original work is properly cited (including links to both the formal publication through the relevant DOI and the license). See: https://creativecommons.org/licenses/by-nc-nd/4.0/.

\section{References}

1. Noble PB, Pascoe CD, Lan B, et al. Airway smooth muscle in asthma: Linking contraction and mechanotransduction to disease pathogenesis and remodelling. Pulm Pharmacol Ther 2014;29:96-107.

2. Reddel HK, Bateman ED, Becker A, et al. A summary of the new gina strategy: A roadmap to asthma control. Eur Respir J 2015;46:622-39.

3. Hirota N, Martin JG. Mechanisms of airway remodeling. Chest 2013;144:1026-32.

4. Halwani R, Al-Muhsen S, Hamid Q. Airway remodeling in asthma. Curr Opin Pharmacol 2010;10:236-45.

5. Bai TR. Evidence for airway remodeling in chronic asthma. Curr Opin Allergy Clin Immunol 2010;10:82-6.

6. Munakata M. Airway remodeling and airway smooth muscle in asthma. Allergol Int 2006;55:235-43.

7. Wright DB, Trian T, Siddiqui S, et al. Phenotype modulation of airway smooth muscle in asthma. Pulm Pharmacol Ther 2013;26:42-9.

8. Qiu C, Zhang J, Su M, et al. Nuclear factor-kappab mediates the phenotype switching of airway smooth muscle cells in a murine asthma model. Int J Clin Exp Pathol 2015;8:12115-28.

9. Qiu C, Liu W, Shi F, et al. Silencing of beta1 integrin regulates airway remodeling by regulating the transcription of soceassociated genes in asthmatic mice. Mol Med Rep 
2017;16:2645-51.

10. Zou JJ, Gao YD, Geng S, et al. Role of stim1/orai1mediated store-operated ca(2)(+) entry in airway smooth muscle cell proliferation. J Appl Physiol (1985) 2011;110:1256263.

11. von Lindern M, van Baal S, Wiegant J, et al. Can, a putative oncogene associated with myeloid leukemogenesis, may be activated by fusion of its 3 ' half to different genes: Characterization of the set gene. Mol Cell Biol 1992;12:3346-55.

12. Switzer CH, Cheng RY, Vitek TM, et al. Targeting set/i(2) pp2a oncoprotein functions as a multi-pathway strategy for cancer therapy. Oncogene 2011;30:2504-13.

13. Mukhopadhyay A, Saddoughi SA, Song P, et al. Direct interaction between the inhibitor 2 and ceramide via sphingolipid-protein binding is involved in the regulation of protein phosphatase $2 \mathrm{a}$ activity and signaling. FASEB J 2009;23:751-63.

14. Janghorban M, Farrell AS, Allen-Petersen BL, et al. Targeting c-myc by antagonizing pp2a inhibitors in breast cancer. Proc Natl Acad Sci U S A 2014;111:9157-62.

15. Sobral LM, Sousa LO, Coletta RD, et al. Stable set knockdown in head and neck squamous cell carcinoma promotes cell invasion and the mesenchymal-like phenotype in vitro, as well as necrosis, cisplatin sensitivity and lymph node metastasis in xenograft tumor models. Mol Cancer 2014;13:32.

16. Fournier PG, Juarez P, Jiang G, et al. The tgf-beta signaling regulator pmepa1 suppresses prostate cancer metastases to bone. Cancer Cell 2015;27:809-21.

17. Basurto-Islas G, Gu JH, Tung YC, et al. Mechanism of tau hyperphosphorylation involving lysosomal enzyme asparagine endopeptidase in a mouse model of brain ischemia. J Alzheimers Dis 2018;63:821-33.

18. Shi F, Qiu C, Qi H, et al. Shrna targeting beta1-integrin suppressed proliferative aspects and migratory properties of airway smooth muscle cells. Mol Cell Biochem 2012;361:111-21.

19. Dekkers BG, Maarsingh H, Meurs H, et al. Airway structural components drive airway smooth muscle remodeling in asthma. Proc Am Thorac Soc 2009;6:683-92.

20. Zhang Q, Giebler HA, Isaacson MK, et al. Eviction of linker histone h1 by nap-family histone chaperones enhances activated transcription. Epigenetics Chromatin 2015;8:30.

21. Eichhorn PJ, Creyghton MP, Bernards R. Protein phosphatase 2a regulatory subunits and cancer. Biochim
Biophys Acta 2009;1795:1-15.

22. Cervoni N, Detich N, Seo SB, et al. The oncoprotein set/taf-1beta, an inhibitor of histone acetyltransferase, inhibits active demethylation of DNA, integrating DNA methylation and transcriptional silencing. J Biol Chem 2002;277:25026-31.

23. Liao Q, Zeng Z, Guo X, et al. Lplunc1 suppresses il6-induced nasopharyngeal carcinoma cell proliferation via inhibiting the stat 3 activation. Oncogene 2014;33:2098-109.

24. Ueki N, Sobue K, Kanda K, et al. Expression of high and low molecular weight caldesmons during phenotypic modulation of smooth muscle cells. Proc Natl Acad Sci U S A 1987;84:9049-53.

25. Halayko AJ, Salari H, Ma X, et al. Markers of airway smooth muscle cell phenotype. Am J Physiol 1996;270:L1040-51.

26. Samanta K, Parekh AB. Store-operated ca2+ channels in airway epithelial cell function and implications for asthma. Philos Trans R Soc Lond B Biol Sci 2016;371:20150424.

27. Chen J, Miller M, Unno H, et al. Orosomucoid-like 3 (ormdl3) upregulates airway smooth muscle proliferation, contraction, and $\mathrm{ca}(2+)$ oscillations in asthma. J Allergy Clin Immunol 2018;142:207-18.e6.

28. Ohga K, Takezawa R, Yoshino T, et al. The suppressive effects of ym-58483/btp-2, a store-operated ca2+ entry blocker, on inflammatory mediator release in vitro and airway responses in vivo. Pulm Pharmacol Ther 2008;21:360-9.

29. Lewis RS. The molecular choreography of a storeoperated calcium channel. Nature 2007;446:284-7.

30. Peel SE, Liu B, Hall IP. Orai and store-operated calcium influx in human airway smooth muscle cells. Am J Respir Cell Mol Biol 2008;38:744-9.

31. Spinelli AM, Gonzalez-Cobos JC, Zhang X, et al. Airway smooth muscle stim1 and orai1 are upregulated in asthmatic mice and mediate pdgf-activated soce, crac currents, proliferation, and migration. Pflugers Arch 2012;464:481-92.

32. Peel SE, Liu B, Hall IP. A key role for stim1 in store operated calcium channel activation in airway smooth muscle. Respir Res 2006;7:119.

33. Plant PJ, North ML, Ward A, et al. Hypertrophic airway smooth muscle mass correlates with increased airway responsiveness in a murine model of asthma. Am J Respir Cell Mol Biol 2012;46:532-40.

34. Vermes I, Haanen C, Steffens-Nakken H, et al. A 
Page 10 of 10

novel assay for apoptosis. Flow cytometric detection of phosphatidylserine expression on early apoptotic cells using fluorescein labelled annexin v. J Immunol Methods

\section{Li et al. SET play an important role in asthma}

1995;184:39-51.

(English Language Editor: C. Betlazar-Maseh)

Cite this article as: Li J, He Q, Wang L, Chen D, Qiu C, Xu P, Lu Y, Zeng Y, Chen R. SET knockdown attenuated phenotype modulation and calcium channel associated markers of airway smooth muscle cells in asthmatic mice. Ann Transl Med 2021;9(8):657. doi: 10.21037/atm-21-573 